\title{
The independence and clique polynomial of the conjugacy class graph of dihedral group
}

\author{
Nabilah Najmuddin a, ${ }^{\star}$, Nor Haniza Sarmin ${ }^{a}$, Ahmad Erfanian ${ }^{\mathrm{b}}$, Hamisan Rahmat a \\ a Department of Mathematical Sciences, Faculty of Science, Universiti Teknologi Malaysia, 81310 UTM Johor Bahru, Johor, Malaysia \\ b Department of Mathematics and Center of Excellence in Analysis on Algebraic Structures, Ferdowsi University of Mashhad, Azadi Square, \\ 9177948974 Mashhad, Razavi Khorasan, Iran \\ *Corresponding author: nabilah.najmuddin@yahoo.com
}

\section{Article history}

Received 3 May 2018

Revised 25 August 2018

Accepted 11 September 2018

Published Online 25 October 2018

\section{Graphical abstract}

Some preliminaries on the independence and clique polynomial as well as the conjugacy class graph are stated.

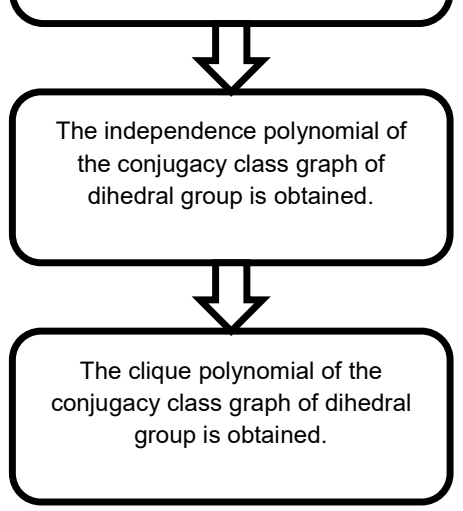

\begin{abstract}
The independence and clique polynomial are two types of graph polynomial that store combinatorial information of a graph. The independence polynomial of a graph is the polynomial in which its coefficients are the number of independent sets in the graph. The independent set of a graph is a set of vertices that are not adjacent. The clique polynomial of a graph is the polynomial in which its coefficients are the number of cliques in the graph. The clique of a graph is a set of vertices that are adjacent. Meanwhile, a graph of group $G$ is called conjugacy class graph if the vertices are noncentral conjugacy classes of $G$ and two distinct vertices are connected if and only if their class cardinalities are not coprime. The independence and clique polynomial of the conjugacy class graph of a group $G$ can be obtained by considering the polynomials of complete graph or polynomials of union of some graphs. In this research, the independence and clique polynomials of the conjugacy class graph of dihedral groups of order $2 n$ are determined based on three cases namely when $n$ is odd, when $n$ and $n / 2$ are even, and when $n$ is even and $n / 2$ is odd. For each case, the results of the independence polynomials are of degree two, one and two, and the results of the clique polynomials are of degree $(n-1) / 2,(n+2) / 2$ and $(n-2) / 2$, respectively.
\end{abstract}

Keywords: Independence polynomial, clique polynomial, conjugacy class graph, dihedral group

\section{INTRODUCTION}

In order to understand this research, few basic concepts from graph theory are first stated here. A graph is $\Gamma=(V, E)$ containing $V$ as a nonempty set of vertices and $E$ as a set of unordered pair of elements of $V$, called the edges. Two vertices $u, v \in V$ are adjacent to each other in $\Gamma$ if and only if there is an edge between $u$ and $v$, i.e. $(u, v) \in E$. A vertex $v$ is an isolated vertex if it is not adjacent to any other vertices $u$ in the graph. An edge $e=(x, y)$ is called incident with each one of its end vertices, $x$ and $y$. Note that, only simple graph is considered throughout this study. A graph is simple if it has no loops and no multiple edges.

A vertex $u$ is a neighbor of vertex $v$ in if $(u, v)$ is an edge of $\Gamma$. Open neighborhood (or just neighborhood), of $v$ is defined to be the set of all vertices adjacent to $v$, denoted as $N(v)=\{u \in V \mid(u, v) \in E, u \neq v\}$. The set $N[v]=N(v) \cup\{v\}$ is the closed neighborhood of $v$ in $\Gamma$ [1]. If the neighborhood of every vertex is empty, means that there is no edge in the graph, then the graph is called empty graph on $n$ vertices, denoted by $E_{n}$. If $n=0$, then the graph is called null graph, denoted by $E_{0}:=\varnothing$, and if we have $n=1$, the graph $E_{1}$ is called singleton, a single vertex graph. The complement of an empty graph is the complete graph, $K_{n}$. It is a graph with $n$ vertices where each pair of distinct vertices is connected by an edge [2].

This paper is structured as follows: the first part is the introduction, the second part is the methodology used in this research while the third part includes the main results. The independence polynomial and clique polynomial are computed for the conjugacy class graph of the dihedral group of order $2 n$, with group representation

$$
D_{2 n}=\left\langle a, b: a^{n}=b^{2}=1, b a b=a^{1}\right\rangle \text {, where } n \geq 3, n \in \square \text {. }
$$




\section{PRELIMINARIES}

Some basic concepts in graph theory that are related to group theory are included in this section. The independence and clique polynomial are determined by using some properties that will be stated here.

The following are some basic concepts on independence polynomial, that are used throughout this paper.

Definition 2.1 [3] Independent Set, Independence Number An independent set of a graph is a set of vertices such that no two distinct vertices are adjacent. The independence number of the graph , denoted by () is the maximum number or vertices in an independent set of the graph.

\section{Definition 2.2 [3] Independence Polynomial}

The independence polynomial of a graph $\Gamma$ is the polynomial whose coefficient on $x^{k}$ is given by the number of independent sets of order $k$ in $\Gamma$. This is denoted by $I(\Gamma ; x)$. So

$$
I(\Gamma ; x)=\sum_{k=0}^{\alpha(\Gamma)} a_{k} x^{k}
$$

where $a_{k}$ is the number of independent sets of size $k$ and ( ) is the independence number of $\Gamma$.

\section{Theorem 2.1 [3]}

Let $\Gamma_{1}$ and $\Gamma_{2}$ be two disjoint graphs. Then

$$
I\left(\Gamma_{1} \cup \Gamma_{2} ; x\right)=I\left(\Gamma_{1} ; x\right) \cdot I\left(\Gamma_{2} ; x\right) .
$$

From the definition and theorem above, Ferrin [4] had applied them in obtaining the independence polynomial of some common graphs. Below are the propositions that will be used in proving our results.

Proposition 2.1 [4]

The independence polynomial of an empty graph on $n$ vertices, is

$$
I\left(E_{n} ; x\right)=I\left(\overline{K_{n}} ; x\right)=(1+x)^{n} .
$$

\section{Proposition 2.2 [4]}

The independence polynomial of a complete graph on $n$ vertices is

$$
I\left(K_{n} ; x\right)=1+n x \text {. }
$$

Next, in the following are the basic concepts on clique polynomial, that will be used throughout this paper.

Definition 2.3 [3] Clique, Clique Number

A clique of a graph is a set of vertices in which every vertex is adjacent to every other vertex. The clique number of the graph , denoted by ( ) is the size of the biggest clique.

Definition 2.4 [3] Clique Polynomial

The clique polynomial of a graph $\Gamma$ is the polynomial whose coefficient on $x^{k}$ is given by the number of cliques of order $k$ in $\Gamma$. This is denoted by $C(\Gamma ; x)$. So

$$
C(\Gamma ; x)=\sum_{k=0}^{\omega(\Gamma)} c_{k} x^{k}
$$

where $c_{k}$ is the number of cliques of order $k$ and $($ ) is the clique number of $\Gamma$.

Theorem 2.2 [3]

Let $\Gamma_{1}$ and $\Gamma_{2}$ be two disjoint graphs. Then

$$
C\left({ }_{1} \quad ; x\right)=C\left({ }_{1} ; x\right)+C\left({ }_{2} ; x\right) 1 .
$$

Hoede and Li [3] had also applied Definition 2.4 and Theorem 2.2 to obtain the following propositions.

\section{Proposition 2.3 [3]}

The clique polynomial of an empty graph on $n$ vertices, is

$$
C\left(E_{n} ; x\right)=C\left(\overline{K_{n}} ; x\right)=1+n x \text {. }
$$

Proposition 2.4 [3]

The clique polynomial of a complete graph on $n$ vertices is

$$
C\left(K_{n} ; x\right)=(1+x)^{n} \text {. }
$$

Lastly, we state some basic definitions and results from group theory and graph theory that are related to conjugacy class graph.

\section{Definition 2.3 [5] Conjugacy Class}

Let $G$ be a group and $a, x \in G$. For a fixed element $a \in G$, the conjugacy class of $a$ in $G$ is given as

$$
c l(a)=\left\{g \in G \text { : there exists } x \in G, g=x_{a x}^{-1}\right\} .
$$

Also, $x_{a x}^{-1}$ is called the conjugate of $a$ by $x$ in the group $G$.

\section{Proposition 2.5 [5]}

Let $a$ be an element in $G$. If the conjugacy class of $a$ contains only one element, then $a$ lies in the center of the group, $Z(G)$.

\section{Theorem 2.3 [6]}

Let $D_{2 n}$ be dihedral groups of order $2 n$, then the conjugacy classes in $D_{2 n}$, depending on the parity of $n$, are as follows.

1. For odd $n$ :

$$
\{1\},\left\{a, a^{1}\right\},\left\{a, a^{2}\right\}, \ldots,\left\{a^{\frac{n 1}{2}}, a^{\frac{(n 1)}{2}}\right\} \text { and }\left\{a^{i} b: 0 \text { i } \quad n \quad 1\right\}
$$

2. For even $n$ :

$$
\begin{aligned}
& \{1\},\left\{a, a^{1}\right\},\left\{a, a^{2}\right\}, \ldots,\left\{a^{\frac{n 2}{2}}, a^{\frac{(n 2)}{2}}\right\},\left\{a^{\frac{n}{2}}\right\}, \\
& \left\{a^{2 i} b: 0 \leq i \leq \frac{n 2}{2}\right\} \text { and }\left\{a^{2 i+1} b: 0 \leq i \leq \frac{n}{2}\right\}
\end{aligned}
$$

Definition 2.4 [7] Conjugacy Class Graph

A conjugacy class graph ${ }_{G}^{c l}$ of a group $G$, is defined as the graph whose vertex set, $V\left(\begin{array}{c}c l \\ G\end{array}\right)$ is non-central conjugacy classes of $G$, in which two distinct vertices $c l(a)$ and $c l(b)$ are adjacent if and only if their class cardinalities are not coprime i.e. $(|\operatorname{cl}(a)|,|c l(b)|)>1$.

\section{Theorem 2.4 [8]}

Let $D_{2 n}$ be dihedral groups of order $2 n$, then the conjugacy class graphs of $D_{2 n}$ can be stated as in the following.

Case 1: $\quad n$ is odd

$$
{ }_{D_{2 n}}^{c l}=K_{\frac{n 1}{2}} \quad K_{1} \text {, such that } K_{\frac{n 1}{2}} \text { contains vertices }
$$
$c l(a), \operatorname{cl}\left(a^{2}\right), \ldots$, and $c l\left(a^{\frac{n 1}{2}}\right)$, and $K_{1}$ is the isolated vertex $c l(b)$.

Case 2: $\quad n$ and $\frac{n}{2}$ are even 
${ }_{D_{2 n}}^{c l}=K_{\frac{n+2}{2}}, \quad$ such that it contains vertices

$c l(a), c l\left(a^{2}\right), \ldots, c l\left(a^{\frac{n 2}{2}}\right), c l(b)$, and $c l(a b)$.

Case 3: $\quad n$ is even and $\frac{n}{2}$ is odd

${ }_{D_{2 n}}^{c l}=K_{\frac{n 2}{2}} \quad K_{2}$, such that $K_{\frac{n 2}{2}}$ contains vertices $c l(a)$,

$c l\left(a^{2}\right), \ldots$, and $c l\left(a^{\frac{n 2}{2}}\right)$, and $K_{2}$ contains vertices $c l(b)$ and $c l(a b)$

The aim of this paper is to obtain the independence and clique polynomial of the conjugacy class graph for the dihedral groups $D_{2 n}$ of order $2 n$.

\section{MAIN RESULTS}

This section consists of two parts. The first part presents the result on the independence polynomial of the conjugacy class graph of $D_{2 n}$ while the second part presents the clique polynomial of the conjugacy class graph of $D_{2 n}$.

\section{The independence polynomial of conjugacy class graph of dihedral groups}

This is the first part of the main result in which the independence polynomials of the conjugacy class graph of $D_{2 n}$ are obtained, depending on the parity of $n$.

Theorem 3.1 Suppose that $D_{2 n}$ be dihedral groups of order $2 n$ where $n \geq 3, n \in \square$ then the independence polynomials of the conjugacy class graphs of $D_{2 n}$ are as follows.

$$
I\left(\begin{array}{ll}
c l \\
D_{2 n}
\end{array} ; x\right)= \begin{cases}1+\left(\frac{n+1}{2}\right) x+\left(\frac{n 1}{2}\right) x^{2} & ; n \text { is odd } \\
1+\left(\frac{n+2}{2}\right) x & ; n \text { and } \frac{n}{2} \text { are even } \\
1+\left(1+\frac{n}{2}\right) x+\left(\begin{array}{ll}
n & 2
\end{array}\right) x^{2} & ; n \text { is even and } \frac{n}{2} \text { is odd }\end{cases}
$$

Proof Let $D_{2 n}$ be dihedral groups of order $2 n$ and ${ }_{D_{2 n}}^{c l}$ be its conjugacy class graph.

Case 1: $n$ is odd

From Theorem 2.4, the conjugacy class graph of $D_{2 n}$ is $K_{\frac{n 1}{2}} K_{1}$ By Theorem 2.1 and Proposition 2.2, we can compute the independence polynomial of $\begin{gathered}c l \\ D_{2 n}\end{gathered}$ as follows:

$$
\begin{aligned}
I\left(\begin{array}{c}
c l \\
D_{2 n}
\end{array} ; x\right) & =I\left(K_{\frac{n 1}{2}} \cup K_{1} ; x\right) \\
& =I\left(K_{\frac{n 1}{2}} ; x\right) \cdot I\left(K_{1} ; x\right) \\
& =\left(1+\left(\frac{n 1}{2}\right) x\right)(1+x) \\
& =1+x+\left(\frac{n 1}{2}\right) x+\left(\frac{n 1}{2}\right) x^{2} \\
& =1+\left(\frac{n+1}{2}\right) x+\left(\frac{n 1}{2}\right) x^{2} .
\end{aligned}
$$

Case 2: $\quad n$ and $\frac{n}{2}$ are even

From Theorem 2.4, the conjugacy class graph of $D_{2 n}$ is $K_{\frac{n+2}{2}}$. By Proposition 2.2, the independence polynomial of ${ }_{D_{2 n}}^{c l}$ can be computed as follows:

$$
\begin{aligned}
I\left(\begin{array}{c}
c l \\
D_{2 n}
\end{array} ; x\right) & =I\left(K_{\frac{n+2}{2}} ; x\right) \\
& =1+\left(\frac{n+2}{2}\right) x .
\end{aligned}
$$

Case 3: $\quad n$ is even and $\frac{n}{2}$ is odd

From Theorem 2.4, the conjugacy class graph of $D_{2 n}$ is $K_{\frac{n 2}{2}} \quad K_{2}$. By Theorem 2.1 and Proposition 2.2, the independence polynomial of ${ }_{D_{2 n}}^{c l}$ can be computed as follows:

$$
\begin{aligned}
I\left(\Gamma_{D_{2 n}}^{c l} ; x\right) & =I\left(K_{\frac{n-2}{2}} \cup K_{2} ; x\right) \\
& =I\left(K_{\frac{n-2}{2}} ; x\right) \cdot I\left(K_{2} ; x\right) \\
& =\left(1+\left(\frac{n-2}{2}\right) x\right)(1+2 x) \\
& =1+2 x+\left(\frac{n-2}{2}\right) x+2\left(\frac{n-2}{2}\right) x^{2} \\
& =1+\left(\frac{2+n}{2}\right) x+(n-2) x^{2} \\
& =1+\left(1+\frac{n}{2}\right) x+(n-2) x^{2} .
\end{aligned}
$$

Example 3.1 Let $G$ be the dihedral group of order $14(n=7)$, i.e. $G=D_{14}=\left\langle a, b: a^{7}=b^{2}=1, b a b=a^{1}\right\rangle$. By Theorem 2.3, the conjugacy classes of $D_{14}$ are $\operatorname{cl}(e), \operatorname{cl}(a)=\left\{a, a^{6}\right\}, \operatorname{cl}\left(a^{2}\right)=\left\{a^{2}, a^{5}\right\}$ $c l\left(a^{3}\right)=\left\{a^{3}, a^{4}\right\} \quad$ and $\quad c l(b)=\left\{b, a b, a^{2} b, a^{3} b, a^{4} b, a^{5} b, a^{6} b\right\} . \quad$ By Theorem 2.4, if $\quad \begin{gathered}c l \\ D_{14}\end{gathered}$ is the conjugacy class graph of $D_{14}$ with the set of vertices, $\quad V\left(\begin{array}{c}c l \\ D_{14}\end{array}\right)=\left\{\operatorname{cl}(a), \operatorname{cl}\left(a^{2}\right), \operatorname{cl}\left(a^{3}\right), \operatorname{cl}(b)\right\}, \quad$ then ${ }_{D_{14}}^{c l}=K_{3} \quad K_{1}$. Hence, the independence polynomial of the conjugacy class graph of $D_{14}$ is

$$
\begin{aligned}
I\left({ }_{D_{14}}^{c l} ; x\right) & =I\left(K_{3} \cup K_{1} ; x\right) \\
& =1+\left(\frac{7+1}{2}\right) x+\left(\frac{71}{2}\right) x^{2} \\
& =1+4 x+3 x^{2} .
\end{aligned}
$$


The clique polynomial of conjugacy class graph of dihedral groups

The second part of our main result is the clique polynomial of the conjugacy class graph of $D_{2 n}$.

Theorem 3.2 Suppose that $D_{2 n}$ be dihedral groups of order $2 n$ where $n \geq 3, n \in \square$ then the clique polynomials of the conjugacy class graphs of $D_{2 n}$ are as follows.

$C\left({ }_{D_{2 n}}^{c l} ; x\right)= \begin{cases}x+(1+x)^{\frac{n 1}{2}} & ; n \text { is odd } \\ (1+x)^{\frac{n+2}{2}} & ; n \text { and } \frac{n}{2} \text { are even } \\ 2 x+x^{2}+(1+x)^{\frac{n 2}{2}} & ; n \text { is even and } \frac{n}{2} \text { is odd. }\end{cases}$

Proof Let $D_{2 n}$ be dihedral groups of order $2 n$ and ${ }_{D_{2 n}}^{c l}$ be its conjugacy class graph.

Case 1: $\quad n$ is odd

From Theorem 2.4, the conjugacy class graph of $D_{2 n}$ is $K_{\frac{n 1}{2}} \quad K_{1}$. By Theorem 2.2 and Proposition 2.4, the clique polynomial of $\quad \begin{gathered}c l \\ D_{2 n}\end{gathered}$ can be computed as follows:

$$
\begin{aligned}
C\left(\begin{array}{c}
c l \\
D_{2 n}
\end{array} ; x\right) & =C\left(K_{\frac{n 1}{2}} \quad K_{1} ; x\right) \\
& =C\left(K_{\frac{n 1}{2}} ; x\right)+C\left(K_{1} ; x\right) \quad 1 \\
& =(1+x)^{\frac{n 1}{2}}+(1+x) \quad 1 \\
& =x+(1+x)^{\frac{n 1}{2}} .
\end{aligned}
$$

Case 2: $n$ and $\frac{n}{2}$ are even

From Theorem 2.4, the conjugacy class graph of $D_{2 n}$ is $K_{\frac{n+2}{2}}$. By Proposition 2.4, the clique polynomial of $\begin{aligned} & c l \\ & D_{2 n}\end{aligned}$ can be obtained as follows:

$$
\begin{aligned}
C\left(\begin{array}{c}
c l \\
D_{2 n}
\end{array} ; x\right) & =C\left(K_{\frac{n+2}{2}} ; x\right) \\
& =(1+x)^{\frac{n+2}{2}} .
\end{aligned}
$$

Case 3: $\quad n$ is even and $\frac{n}{2}$ is odd

From Theorem 2.4, the conjugacy class graph of $D_{2 n}$ is $K_{\frac{n 2}{2}} \quad K_{2}$. By Theorem 2.2 and Proposition 2.4, the clique polynomial of $\quad \begin{gathered}c l \\ D_{2 n}\end{gathered}$ can be computed as follows:

$$
\begin{aligned}
C\left(\Gamma_{D_{2 n}}^{c l} ; x\right) & =C\left(K_{\frac{n-2}{2}} \cup K_{2} ; x\right) \\
& =C\left(K_{\frac{n-2}{2}} ; x\right)+C\left(K_{2} ; x\right)-1 \\
& =(1+x)^{\frac{n-2}{2}}+(1+x)^{2}-1 \\
& =(1+x)^{\frac{n-2}{2}}+1+2 x+x^{2}-1 \\
& =2 x+x^{2}+(1+x)^{\frac{n-2}{2}} .
\end{aligned}
$$

Example 3.2 Let $G$ be the dihedral group of order $12(n=6)$, i.e. $G=D_{12}=\left\langle a, b: a^{6}=b^{2}=1, b a b=a^{1}\right\rangle$. By Theorem 2.3, the conjugacy classes of $D_{12}$ are $\operatorname{cl}(e), \operatorname{cl}(a)=\left\{a, a^{5}\right\}, \operatorname{cl}\left(a^{2}\right)=\left\{a^{2}, a^{4}\right\}$ $c l\left(a^{3}\right), \quad c l(b)=\left\{b, a^{2} b, a^{4} b\right\} \quad$ and $\quad c l(a b)=\left\{a b, a^{3} b, a^{5} b\right\} . \quad$ Вy Theorem 2.4, if $\Gamma_{D_{12}}^{c l}$ is the conjugacy class graph of $D_{14}$ with the set of vertices, $\quad V\left(\Gamma_{D_{12}}^{c l}\right)=\left\{\operatorname{cl}(a), \operatorname{cl}\left(a^{2}\right), \operatorname{cl}(b), \operatorname{cl}(a b)\right\}, \quad$ then $\Gamma_{D_{12}}^{c l}=K_{2} \cup K_{2}$. Hence, the clique polynomial of the conjugacy class graph of $D_{12}$ is

$$
\begin{aligned}
C\left(\Gamma_{D_{12}}^{c l} ; x\right) & =C\left(K_{2} \cup K_{2} ; x\right) \\
& =2 x+x^{2}+(1+x)^{2} \\
& =2 x+x^{2}+1+2 x+x^{2} \\
& =1+4 x+2 x^{2} .
\end{aligned}
$$

\section{CONCLUSION}

In this paper, the independence polynomial and clique polynomial of the conjugacy class graph of the group $D_{2 n}$ are obtained. The results are based on three cases which are when $n$ is odd, when $n$ and $\frac{n}{2}$ are even, and also when $n$ is even and $\frac{n}{2}$ is odd. The independence polynomial of the conjugacy class graph of $D_{2 n}$ is $I\left(\begin{array}{c}c l \\ D_{2 n}\end{array} ; x\right)=1+\left(\frac{n+1}{2}\right) x+\left(\frac{n}{2}\right) x^{2}$ when $n$ is odd, $I\left(\begin{array}{c}c l \\ D_{2 n}\end{array} ; x\right)=1+\left(\frac{n+2}{2}\right) x$ when $n$ and $\frac{n}{2} \quad$ are even, and $I\left(\begin{array}{c}c l \\ D_{2 n}\end{array} ; x\right)=1+\left(1+\frac{n}{2}\right) x+\left(\begin{array}{ll}n & 2\end{array}\right) x^{2}$ when $n$ is even and $\frac{n}{2}$ is odd. Meanwhile, the clique polynomial of the conjugacy class graph of $D_{2 n}$ is $C\left(\begin{array}{c}c l \\ D_{2 n}\end{array} ; x\right)=x+(1+x)^{\frac{n 1}{2}}$ when $n$ is odd. When $n$ and $\frac{n}{2}$ are even, $C\left(\begin{array}{c}c l \\ D_{2 n}\end{array} ; x\right)=(1+x)^{\frac{n+2}{2}}$ and when $n$ is even and $\frac{n}{2}$ is odd, $C\left(\begin{array}{c}c l \\ D_{2 n}\end{array} ; x\right)=2 x+x^{2}+(1+x)^{\frac{n 2}{2}}$.

\section{ACKNOWLEDGEMENT}

The first author would like to thank Universiti Sains Malaysia and the Ministry of Higher Education for the full scholarship given. This work was financially supported by the Universiti Teknologi Malaysia and Ministry of Higher Education under the Research University Grant (GUP) Vote No 13J82. 


\section{REFERENCES}

[1] Balakrishnan, R. and Ranganathan, K. 2012. A Textbook of Graph Theory. $2^{\text {nd }}$ ed. New York: Springer.

[2] Rosen, K. H. 2013. Discrete Mathematics and Its Applications. $7^{\text {th }}$ ed. New York: McGraw-Hill

[3] Hoede, C. and Li, X. 1994. Clique Polynomials and Independent Set Polynomials of Graphs. Discrete Mathematics. 125: 219-228.

[4] Ferrin, G. 2014. Independence Polynomials. Master Dissertation. University of California.

[5] Harvey, E. R. 2009. A Course in Finite Groups. London: SpringerVerlag.

[6] Samaila, D., Abba, B. I. and Pur, M. P.. 2013. On the conjugacy classes, centers and representation of the groups $S_{n}$ and $D_{n}$. International Journal of Pure and Applied Science and Technology. 15(1): 87-95.

[7] Bertram, E. A., Herzog, M. and Mann, A. 1990. On a graph related to conjugacy classes of groups. Bulletin of the London Mathematical Society. 22(6): 569-575.

[8] Mahmoud, R., Sarmin, N. H. and Erfanian, A. 2017. The conjugacy class graph of some finite groups and its energy. Malaysian Journal of Fundamental and Applied Sciences. 13(4): 659-665.

[9] Omer, S. M. S., Sarmin, N. H. and Erfanian, A.. 2013. The probability that an element of a group fixes a set and its graph related to conjugacy classes. Journal of Basic Applied Scientific Research. 3(10): 369-380.

[10] Sarmin, N. H., Gambo, I. and Omer, S. M. S. 2015. The conjugacy classes of metabelian groups of order at most 24. Jurnal Teknologi 77(1) : 139-143.

[11] Sarmin, N. H., Bilhikmah, N. H., Omer, S. M. S. and Noor, A. H. M. 2016. The conjugacy classes, conjugate graph and conjugacy class graph of some finite metacyclic 2-groups. Menemui Matematik. 38(1): 1-12. 